SOBRE LA SUSTENTABILIDAD DESDE LA UNIVERSIDAD, UN PROYECTO DE CONSULTORÍA EN RELACIONES INDUSTRIALES

\title{
SOBRE LA SUSTENTABILIDAD DESDE LA UNIVERSIDAD, UN PROYECTO DE CONSULTORÍA EN RELACIONES INDUSTRIALES
}

García Panes Luz María*, Alonso Ramírez Karina **

*Maestra en Educación. Universidad Veracruzana.

**Maestra en Administración Pública. Universidad Veracruzana.

Dirección para recibir correspondencia: luz_og@hotmail.com 


\section{RESUMEN}

La gestión del capital humano significa un reto para los directivos, se puede decir que es tan importante como compleja, ya que requiere de conocimientos especializados para elaborar estrategias enfocadas al desarrollo, permanencia y motivación de los individuos dentro de una organización. La facultad de Ciencias Administrativas y Sociales de la Universidad Veracruzana, mediante sus trabajos de vinculación, entiende la necesidad de los empresarios de la región y nace el proyecto para la implementación de una consultoría que brinde servicios de apoyo para la gestión del recurso humano, involucrando a las organizaciones en temas novedosos para mejorar el desempeño de sus trabajadores, promover un buen ambiente de trabajo así como, conservar un alto nivel de motivación y con ello mejorar la productividad y posicionamiento en el mercado de las empresas veracruzanas.

Palabras clave: Capital humano. Consultoría. Emprendimiento social. 


\section{ABSTRACT}

The administration of human capital is a challenge for the managers, it could be said it is as important as complex since it requires specialized knowledge for developing strategies focused on development, permanence and motivation of the people on an organization. The faculty of Administrative and Social Sciences through its liaison work,

understands the needs of the region's businessman in the region, so they create a project for the implementation of a consultancy which provides services for the support of the management of human resources, involving the organizations in novelty themes to improve their workers performance, promote a good job environment, and preserves a high level of motivation, improving the productivity and positioning in the market of the business of Veracruz

Key words: Human capital. Consulting. Social entrepreneurship. 
SOBRE LA SUSTENTABILIDAD DESDE LA UNIVERSIDAD, UN PROYECTO DE CONSULTORÍA EN RELACIONES INDUSTRIALES

\section{Introducción}

Las organizaciones se integran por recursos varios, tales como: financieros, materiales, tecnológicos, pero sin duda el más importante es el recurso humano, que con sus múltiples conocimientos, habilidades, experiencia y actitudes entrelaza los demás para el éxito de las institucionales.

El capital humano representa el soporte y motor de toda organización, su influencia es decisiva para la evolución, crecimiento y futuro de la misma. El hombre es y seguirá siendo el recurso más valioso de una institución.

El término capital humano es utilizado cuando se hace referencia a las capacidades que adquieren las personas con el entrenamiento, capacitación y experiencia, es decir, a las habilidades adquiridas y las capacidades desarrolladas de un individuo a través de la "inversión" que realizan las organizaciones para incrementar la productividad.

La competitividad que existe en el mercado hace indispensable la gestión del capital humano a través de estrategias que aseguren a la organización contar con el personal calificado y competente para la actividad que desempeña, fomentar su desarrollo y dinamismo en el área de trabajo para preservar un buen clima laboral. Así mismo, la planeación y organización del recurso humano deben estar alineados a la cultura organizacional para el logro de sus objetivos.

El presente proyecto, describe la implementación de una consultoría en la Facultad de Ciencias Administrativas y Sociales, con la finalidad de compartir con el sector empresarial tanto público como privado, el talento de los académicos y brindar asesoría para el desarrollo de su personal, fomentando la capacitación de calidad, diseño e implementación de estrategias para la retención de talentos, promover un ambiente laboral sano, así como la elaboración de manuales que propicien la ejecución de tareas de manera ordenada, en el tiempo justo, promoviendo las mejores prácticas para la gestión de personal y en consecuencia la productividad empresarial. 
Nace a partir de la instalación del Aula de Capacitación en la Facultad de Ciencias Administrativas y Sociales de la UV, ante la necesidad de ampliar y aprovechar las capacidades de los especialistas en las áreas del programa educativo de Relaciones Industriales y con el firme compromiso de fortalecer y apoyar el desarrollo económico y productivo de nuestro Estado.

Veracruz se caracteriza por tener municipios ricos en recursos naturales en donde se elaboran productos que son apreciados en otros estados y países, así como servicios privados y trámites administrativos desde el sector público.

De aquí que surjan diversas organizaciones tanto privadas como del sector público que se encargan de la elaboración de productos y servicios, sin embargo, la mayoría de las empresas regionales cuentan con una organización familiar en donde el mando por lo regular lo tienen los jefes de familia, que son los que se encargan de proveer a la empresa de todos los recursos necesarios para la producción, dejando de lado cuestiones tan importantes como la administración y estructuración de la organización. Este plan surge como una actividad estratégica de vinculación cuyo propósito es, fortalecer y apoyar a las organizaciones de Xalapa y sus alrededores elevando su competitividad y productividad.

El proyecto articula la intervención profesional de los académicos al sector empresarial, apoyados en su momento por estudiantes, los cuales se insertan en organizaciones de la región.

Esta relación permanente y sistemática, permite conocer las necesidades y requerimientos de los empresarios, para enfocarse en sus áreas de oportunidad y ofrecerles estrategias de mejora que impactarán positivamente en la productividad, con lo cual se logrará abrir una nueva puerta hacía el sector productivo a los egresados de $\mathrm{RI}$, con grandes beneficios para las empresas veracruzanas, lo cual se traducirá en un bien social. 
SOBRE LA SUSTENTABILIDAD DESDE LA UNIVERSIDAD, UN PROYECTO DE CONSULTORÍA EN RELACIONES INDUSTRIALES

\section{Marco teórico}

La consultoría es un servicio de asesoramiento independiente que ayuda a los directivos a alcanzar los objetivos, (Sandoval, 2015). Como campo de acción ofrece un sin fin de oportunidades para desarrollarnos profesionalmente y lograr ser agentes de cambio para la mejora continua de las organizaciones y los miembros de la misma; y si existe una profesión que tenga elementos para evolucionar en el ámbito de la consultoría, ésta es la Licenciatura en Relaciones Industriales, porque su formación está enfocada para apoyar a otras personas y a su vez ayudarlas a desarrollar sus capacidades para desenvolverse satisfactoriamente en los distintos ámbitos laborales y llegar a ser competentes.

Encontramos diversas definiciones de consultoría, sin embargo, para efectos del presente proyecto se considerará la siguiente:

"La consultaría de empresas es un servicio de asesoramiento profesional independiente que ayuda a los gerentes y a las organizaciones a alcanzar los objetivos y fines de la organización mediante la solución de problemas gerenciales y empresariales, el descubrimiento y la evaluación de nuevas oportunidades, el mejoramiento del aprendizaje y la puesta en práctica de cambios". (Kubr, 1997, p. 7).

Es relevante conocer las características principales de una consultoría, con el fin de tener claro el funcionamiento y desarrollo de la misma, ya que muchas veces las organizaciones desconocen por completo la metodología y los beneficios, y es por esto que, desgraciadamente pocas son las empresas que acuden con expertos que puedan orientarlos.

"Algunos rasgos que caracterizan a una consultoría son:

$\checkmark$ La consultoría es solo un servicio consultivo. Los consultores son contratados como asesores, que poseen una cierta responsabilidad de la calidad de su consejo, el cuál debe ser apropiado y oportuno. 
$\checkmark$ La consultoría es un servicio profesional en el cual se proporcionan conocimientos y capacidades profesionales para la resolución de problemas que a menudo presentan la dirección de una empresa.

$\checkmark$ La consultoría no proporciona soluciones milagrosas, es más bien una actividad basada en los hechos de una compañía y en la búsqueda de soluciones originales y factibles". (Kubr, 1997, p. 5).

Muchos son los beneficios que se logran a través de una consultoría y es tarea de ésta hacerle ver al cliente que más que un gasto es una inversión a corto y largo plazo que se verá reflejado en la productividad, en el bienestar de sus miembros y por consiguiente el éxito de la empresa.

Entre los beneficios más importantes encontramos los siguientes:

$\checkmark$ El cliente resuelve con el apoyo del consultor el problema específico que enfrenta.

$\checkmark$ El cliente aprende a enfrentarse más acertadamente en el futuro al o a los problemas con los que inició el proceso de consultoría.

$\checkmark$ El cliente aprende nuevos mecanismos de coordinación y adaptación que le ayudan a mantener una salud organizacional estable y le facilita la realización de cambios sin dejar de ser productivo.

$\checkmark$ La empresa se auto regenera a través de la mejora continua lograda por la aplicación de los mecanismos y técnicas definidas en conjunto con el consultor.

$\checkmark$ La empresa se prepara para recibir los cambios que vienen aparejados con los procesos de crecimiento de ésta en el mercado local o foráneo.

\section{METODOLOGÍA}

\section{Objetivo general}

Ofrecer servicios profesionales para contribuir a las necesidades administrativasoperativas y sociales que enfrentan las organizaciones públicas y privadas del Estado de Veracruz. 
SOBRE LA SUSTENTABILIDAD DESDE LA UNIVERSIDAD, UN PROYECTO DE CONSULTORÍA EN RELACIONES INDUSTRIALES

\section{Objetivos específicos}

- Vincular a la facultad con las organizaciones del orden público y privado.

- Mantener la actualización del personal docente con las realidades del campo laboral de la región.

- Establecer un vínculo de comunicación entre la facultad y los empresarios.

- Consolidar el trabajo de equipo entre la comunidad del programa educativo de RI.

- Ser un enlace entre los estudiantes que egresan y el campo laboral.

- Convertir a la facultad en un agente de cambio hacia el sector empresarial xalapeño, de la región y del Estado de Veracruz.

- Apoyar el crecimiento y proyección de las empresas a través de una labor de consultoría específica de acuerdo a las necesidades de las organizaciones.

\section{Estructura de la consultoría}

\section{Misión}

Ofrecer a las empresas soluciones para el mantenimiento y desarrollo de su competitividad y efectividad, así mismo, ofrecer asesoría y apoyo mediante sus servicios profesionales de Recursos Humanos reconocidos por su alta calidad, adecuación y accesibilidad.

\section{Visión}

Proyectarnos como una sólida opción consultora y de gestión, acorde a las necesidades específicas y actuales del sector laboral y el contexto productivo de las organizaciones de la región y del estado de Veracruz.

\section{Objetivo}

Grupo ASECORI está comprometido en desarrollar propuestas y soluciones integrales en Recursos Humanos en las complejas demandas de las organizaciones en los tiempos actuales, caracterizados por una competencia intensa y demandas cada vez más exigentes del mercado. 
Gestionar procesos elaborados a la medida de las necesidades de cada empresa asegurando su calidad y éxito.

1. Brindar asesoría, servicios y sistemas de alta calidad, que permitan proporcionar un verdadero y eficiente soporte a las organizaciones, logrando así su creciente desarrollo y su autonomía.

2. Establecer procesos de capacitación, administración de personal y consultoría que ayude con el desarrollo profesional del capital humano que integra la empresa.

\section{Valores}

- Autodominio. Es el valor que nos ayuda a controlar los impulsos de nuestro carácter y la tendencia a la comodidad mediante la voluntad. Nos estimula a afrontar con serenidad los contratiempos y a tener paciencia y comprensión en las relaciones personales.

- Objetividad. Es el valor de ver el mundo como es, y no como queremos que sea. Los seres humanos somos una compleja mezcla de sentimientos, raciocinio, experiencia y aprendizaje. Todos estos elementos pueden brindar a una persona una percepción de la realidad que puede estar equivocada.

- Puntualidad. El valor que se construye por el esfuerzo de estar a tiempo en el lugar adecuado. Es necesario para dotar a nuestra personalidad de carácter, orden y eficacia, pues al vivir este valor en plenitud estamos en condiciones de realizar más actividades, desempeñar mejor nuestro trabajo, ser merecedores de confianza.

La falta de puntualidad habla por sí misma, de ahí se deduce con facilidad la escasa o nula organización de nuestro tiempo.

- Flexibilidad. Se refiere a la capacidad de adaptarse rápidamente a las circunstancias, los tiempos y las personas, rectificando oportunamente nuestras actitudes y puntos de vista para lograr una mejor convivencia y entendimiento con los demás.

- Crítica Constructiva. El valor de la crítica constructiva se fundamenta en el propósito de lograr un cambio favorable que beneficie a todas y cada una de las personas 
involucradas en circunstancias o ambientes determinados, con actitud de respeto y sentido de colaboración.

- Servicio. Brindar ayuda de manera espontánea en los detalles más pequeños, habla de nuestro alto sentido de colaboración para hacer la vida más ligera a los demás.

- Superación. Es el valor que motiva a la persona a perfeccionarse a sí misma, en lo humano, espiritual, profesional y económico, venciendo los obstáculos y dificultades que se presenten, desarrollando la capacidad de hacer mayores esfuerzos para lograr cada objetivo que se proponga.

- Voluntad. Es la capacidad de los seres humanos que nos mueve a hacer cosas de manera intencionada, por encima de las dificultades, los contratiempos y el estado de ánimo. Es el motor de los demás valores, no sólo para adquirirlos sino para perfeccionarlos, ningún valor puede cultivarse por sí solo si no hacemos un esfuerzo, pues todo requiere pequeños y grandes sacrificios realizados con constancia.

- Compromiso. Una persona comprometida es aquella que cumple con sus obligaciones haciendo un poco más de lo esperado al grado de sorprendernos, porque vive, piensa y sueña con sacar adelante a su familia, su trabajo, su estudio, entre otros.

- Respeto. Es establecer hasta donde llegan mis posibilidades de hacer o no hacer, y dónde comienzan las posibilidades de los demás. El respeto es la base de toda convivencia en sociedad.

- Autenticidad. Las experiencias, el conocimiento y la lucha por concretar propósitos de mejora, hacen que con el tiempo se vaya conformando una personalidad propia El valor de la autenticidad le da a la persona autoridad sobre sí mismo ante sus gustos y caprichos, iniciativa para proponerse y alcanzar metas altas, carácter estable y sinceridad a toda prueba, lo que le hace tener una coherencia de vida.

- Honestidad. Es una forma de vivir congruente entre lo que se piensa y la conducta que se observa hacia el prójimo, que, junto a la justicia, exige en dar a cada quien lo que le es debido. 
- Empatía. La empatía es el esfuerzo que realizamos para reconocer y comprender los sentimientos y actitudes de las personas, así como las circunstancias que los afectan en un momento determinado.

\section{Filosofía}

Generar valor a nuestros clientes con responsabilidad y compromiso mediante el impulso de la profesionalización y la competitividad global, manteniendo el trabajo en equipo y un excelente ambiente de trabajo y así mismo, ser una fuente generadora de empleos para el país.

\section{Principios}

Su filosofía de trabajo se basa en los siguientes principios:

- En sus contratos de Consultoría asume el compromiso de encontrar la solución definitiva para las necesidades de su Cliente y de los Recursos Humanos involucrados.

- Inicia cada proyecto de Consultoría invirtiendo su experiencia profesional en el más completo conocimiento de su Empresa Cliente y en los Recursos Humanos asesorados.

- Su relación con los Clientes y los Recursos Humanos es de alto compromiso profesional con la ética y objetivos institucionales.

- Toda la información sobre clientes se mantiene bajo la más estricta reserva profesional, salvo que ambos autoricen a revelarla.

\section{Metas}

\section{A corto plazo}

1. Ofertar y promocionar un portafolio concreto de opciones en servicios publicitarios y de relaciones públicas, de recursos humanos y de negocios internacionales que permitan proyectar nuestra competitividad y calidad.

2. Al mismo tiempo, conformar un equipo de profesionales que den base a un proyecto autofinanciable de FCAS, en el área en las que somos especialistas. 
SOBRE LA SUSTENTABILIDAD DESDE LA UNIVERSIDAD, UN PROYECTO DE CONSULTORÍA EN RELACIONES INDUSTRIALES

\section{A mediano plazo}

1. Consolidar el portafolio de servicios y ampliar las opciones dentro del mismo, enriqueciéndolo conforme a las necesidades demandadas por el contexto local, la competitividad empresarial y de servicios del entorno inmediato.

2. Así mismo, fortalecer y ampliar redes de profesionales que diversifiquen las opciones y consolidación del proyecto.

\section{Participantes en el proyecto}

Nuestro equipo de trabajo para este proyecto está compuesto por catedráticos de FCAS, profesionales que cuentan con el conocimiento, las habilidades y la actitud necesarias para desarrollar un trabajo de principio a fin; tratando siempre de exceder las expectativas de nuestros clientes y dar solución a sus necesidades, buscando incidir en el crecimiento del sector productivo y público como agentes impulsores del desarrollo de Veracruz.

Cada uno de estos actores participantes de la consultoría, incluirá de acuerdo a su especialidad, los servicios con los que actualmente se pueda comprometer, e invitará a participar a los compañeros profesionales con los que comparta esas áreas, temas o habilidades, y quieran formar parte del equipo de trabajo de profesionistas.

\section{Logística de la Consultoría}

\section{Primera etapa.}

1. La coordinación de vinculación contactará a los empresarios o encargados de las secretarias del sector privado, con la finalidad de explicarles y darles a conocer nuestro nuevo servicio de consultoría y los productos con los que cuenta. En el lapso de mayo a agosto de 2016, se debe haber citado a la mayor cantidad de empresarios xalapeñas así como organizaciones de la secundaria, para iniciar las actividades tan pronto como sea posible.

\section{Segunda etapa.}

2. La coordinación de vinculación canalizará las solicitudes del empresario a la dirección de la escuela. 
3. La dirección de la escuela, se contactará con la encargada del aula de capacitación, para hacerle llegar las necesidades de los empresarios que ASECORI es capaz de atender. Esto se hará lo más apegado a la realidad posible, tratando de que nuestra consultoría se distinga por su formalidad, en cuánto a entrega y calidad en el servicio y/o producto.

4. La encargada del aula de capacitación se entrevistará con los especialistas involucrados en esa área, tema o servicio de que se trate, para conocer si podrá participar en ese momento, de tal manera que se empiece a trabajar de acuerdo con la programación acordada con el empresario.

5. Cuando se llegue a finalizar la gestión consultora, se deberá hacer un seguimiento y evaluación, esto a manera de tener una retroalimentación y conocer el grado de satisfacción del empresario. En caso de que se localice alguna anomalía o insatisfacción por parte de la empresa que contrató el servicio y/o producto, se deberá resolver de inmediato.

\section{Productos y servicios con los que iniciará operaciones}

Diagnóstico Organizacional

Capacitación de Personal

- Desarrollo de habilidades directivas

- Liderazgo

- Plan de vida y carrera

- Honestidad y valores

- Motivación del recurso humano

- Manejo de conflictos laborales

- Trabajo colaborativo

- Prevención de riesgos de trabajo

- Taller de manejo del estrés

- Desarrollo de inteligencia emocional

- Administración del tiempo como herramienta de desempeño eficaz

- Asertividad 
SOBRE LA SUSTENTABILIDAD DESDE LA UNIVERSIDAD, UN PROYECTO DE CONSULTORÍA EN RELACIONES INDUSTRIALES

- Habilidades del pensamiento

- Diseño de estructura y reingeniería de procesos

- Detección de análisis de necesidades de adiestramiento, capacitación y desarrollo

- Evaluación del desempeño personal

- Formación y desarrollo de equipos de trabajo

- Reclutamiento y selección de personal

- Administración efectiva en recursos humanos

- Organización de juntas efectivas

- Taller de técnicas para hablar en público

- Taller de manejo del estrés

- Integridad como estrategia para ser rentable

- Calidad total

- Habilidades del pensamiento

- Salud ocupacional

- Gestión del capital humano por competencias laborales

Planeación estratégica

Diseño de un programa motivacional

Manual de seguridad e higiene

Reclutamiento de Personal

Selección de Personal

Psicodiagnóstico Laboral

Evaluación de personal

Nota: Los precios se ajustarán de acuerdo a los requerimientos de cada empresa.

\section{RESULTADOS}

La consultoría se encuentra en este momento en su etapa inicial, como casos de éxito tenemos las vinculaciones que se realizan a través de las experiencias educativas que brindan servicio a diversas empresas de la región y al sector público. El año pasado se 
SOBRE LA SUSTENTABILIDAD DESDE LA UNIVERSIDAD, UN PROYECTO DE CONSULTORÍA EN RELACIONES INDUSTRIALES

realizaron un promedio de cincuenta vinculaciones en las que se ofrecieron entre otros servicios manuales de motivación, manual de reingeniería, cursos de capacitación, diagnósticos organizacionales, programas de salud ocupacional y manuales de seguridad e higiene.

Como servicio directo se atendió la demanda de la empresa Proinvel la cual estableció contacto con la consultoría, solicitando la atención de sus necesidades de 140 colaboradores, entre los servicios prestados fueron un manual de seguridad e higiene, cursos de capacitación en comunicación y trabajo en equipo, diseño de puesto y evaluación de personal, este proyecto fue exitoso ya que se realizó desde el diagnostico de necesidades hasta el seguimiento de resultados.

Otro de los servicios que se ofrecieron fueron a la empresa Centauro con la que se atendió a un total de 50 colaboradores en un curso de capacitación sobre motivación laboral, estos cursos fueron realizados en la Facultad de Ciencias Administrativas y Sociales.

Reconocer la importancia del recurso humano para el buen desempeño de las organizaciones ha llevado a las empresas a la innovación de estrategias que permiten la provisión de personal competente, así como su crecimiento y retención dentro de la estructura organizacional. De acuerdo a lo anterior, los servicios que ofrece ASECORI se encaminan al mejoramiento de la gestión del capital humano, comprometiéndose con la cultura organizacional para la implementación de guías de acción que permitan al personal conducirse de manera eficiente mejorando su desempeño y productividad organizacional.

ASECORI, valora el poder que el personal ejerce para el cumplimiento de los objetivos en cada institución, es por ellos que los servicios que ofrece se encuentran fuertemente vinculados con la competitividad del personal en el mercado laboral y con ello promover el crecimiento organizacional. 
Con esta consultoría se pretende que la Facultad de Ciencias Administrativas y Sociales de la Universidad Veracruzana, concretamente el programa educativo de Relaciones Industriales ingrese al mercado laboral de las consultorías en recursos humanos de la región de Xalapa, ofreciendo por un lado servicios a las empresas y al sector público de asesoría en la administración del capital humano de calidad y por otra parte pretende ser un vínculo entre las empresas y la oferta de trabajo para los egresados del programa educativo de relaciones industriales, de esta manera se da a conocer la licenciatura y se abren las oportunidades para los egresados.

Finalmente, es una manera de que la Facultad de Ciencias Administrativas y Sociales obtenga sus propios recursos para que en la medida de lo posible sea sustentable. 
SOBRE LA SUSTENTABILIDAD DESDE LA UNIVERSIDAD, UN PROYECTO DE CONSULTORÍA EN RELACIONES INDUSTRIALES

\section{REFERENCIAS BIBLIOGRÁFICAS}

Kubr, M. (1997). La consultoría de empresas: guía para la profesión. Ginebra: OIT.

Robbins, Stephen. (2013). Comportamiento organizacional. México: Prentice Hall.

Sandoval, Suset (2015). Consultoría Empresarial. Recuperado de http://www.tress.com.mx/Boletin/Febrero2015/articulo2.html 